Participants who maintained adequate vitamin D levels over 5 years had significantly less WOMAC knee pain ( $\beta$ :-38.4, 95\% Cl:-69.2,-7.7) and physical dysfunction ( $\beta$ :-98.5, 95\% Cl:-193.8,-3.1) than participants with vitamin D deficiency over 5 years in multivariable analyses.

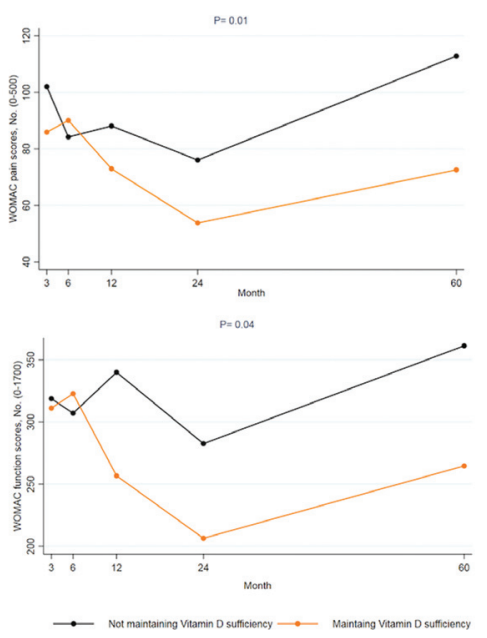

Conclusions: Vitamin D supplementation over 2 years did not result in significant differences in change in knee symptom score over 5 years compared to placebo. However, knee OA patients maintaining sufficient serum vitamin D levels over long-term had more improvement in knee pain and physical function than those who did not maintain adequate vitamin $D$ levels, suggesting a beneficial effect of maintaining sufficient serum vitamin $D$ for knee $O A$.

Disclosure of Interest: None declared

DOI: 10.1136/annrheumdis-2018-eular.4367

\section{FRI0529 PREOPERATIVE PAIN SEEMS TO MODIFY THE EFFECT OF RADIOGRAPHIC OSTEOARTHIRITIS SEVERITY ON POSTOPERATIVE PAIN AND FUNCTION 1 YEAR AFTER TOTAL KNEE ARTHROPLASTY}

R. van de Water ${ }^{1}$, C. Leichtenberg ${ }^{1}$, R. Nelissen ${ }^{1}$, H. Kroon ${ }^{2}$, H. Kaptijn ${ }^{3}$, R. Onstenk ${ }^{4}$, S. Verdegaal ${ }^{5}$, T. Vliet Vlieland ${ }^{1}$, M. Gademan ${ }^{1,6}$ on behalf of the LOAS Study group. ${ }^{1}$ Department of Orthopaedics; ${ }^{2}$ Department of Radiology, LUMC, Leiden; ${ }^{3}$ Department of Orthopaedics, Lange Land Hospital, Zoetermeer, ${ }^{4}$ Department of Orthopaedics, Groene Hart Hospital, Gouda; ${ }^{5}$ Department of Orthopaedics, Alrijne Hospital, Leiderdorp; ${ }^{6}$ Department of Clinical Epidemiology, LUMC, Leiden, Netherlands

Background: Radiographic osteoarthritis (OA) severity and pain play an important role in the indication for total knee arthroplasty (TKA). It is however unkonw whether preoperative self-reported clinical pain modifies the effect of radiographic OA severity on postoperative pain and function in OA patients after TKA.

Objectives: To investigate whether preoperative self-reported clinical pain modified the effect of radiographic OA severity on postoperative pain and function in OA patients 1 year after TKA.

Methods: Data from the Longitudinal Leiden Orthopaedics Outcomes of Osteoarthritis Study (LOAS), a multicentre cohort study on outcomes after TKA were used. Radiographic OA severity was assessed with the Kellgren and Lawrence $(\mathrm{KL})$ score (0-4). Pain and function were evaluated with the Knee Injury and Osteoarthritis Outcome Score (KOOS). After adjustment for confounders (BMI, age, gender and Mental Health Component Scores from the Short Form-12), multivariate linear regression analyses with an interaction term between $\mathrm{KL}$-score and preoperative pain were performed.

Results: 560 patients were included. Both $\mathrm{KL}$-score and preoperative pain were associated with postoperative pain $(\beta: 6.1,95 \% \mathrm{Cl}: 1.7-10.5$ and $\beta: 0.4,95 \% \mathrm{Cl}$ $0.1-0.7))$ and function $(\beta: 8.0,95 \% \mathrm{Cl}: 3.7-12.3$ and $\beta 0.5,95 \% \mathrm{Cl} 0.2-0.8)$. A trend towards effect modification of preoperative pain on the association between $\mathrm{KL}$-score and postoperative pain $(\beta-0.1,95 \% \mathrm{Cl}-0.2-0.0)$ and function $(\beta-0.1$, $95 \% \mathrm{Cl}-0.2-0.0$ ) was found indicating that effect of preoperative pain on postoperative pain and function seems to become less important when more severe radiographic severity is present.
Conclusions: Patients with less preoperative pain and higher $\mathrm{KL}$ grades have better function and pain outcomes 12 months after TKA. However preoperative pain seems less importance in patients with more severe radiographic $O A$.

Acknowledgements: This study was supported by the Dutch Arthritis Association [grant number LLP13].

Disclosure of Interest: None declared

DOI: 10.1136/annrheumdis-2018-eular.3474

\section{FRI0530 ENDPOINT IN OA; PREVALENCE AND INCIDENCE RATES OF TJRS FROM THE PROSPECTIVE EPIDEMIOLOGIC RISK FACTOR (PERF I) STUDY}

A.C. Bay-Jensen ${ }^{1}$, C. Bager ${ }^{1}$, A. Bihlet ${ }^{2}$, C.S. Thudium ${ }^{1}$, I. Byrjalsen ${ }^{2}$, H. B. Nielsen ${ }^{2}$, J.R. Andersen ${ }^{2}$, B.J. Riis ${ }^{2}$, C. Christiansen ${ }^{2}$, M.A. Karsdal ${ }^{1}$. ${ }^{1}$ Rheumatology, Nordic Bioscience Biomarkers and Research; ${ }^{2}$ Clinical Development, Nordic Bioscience, Herlev, Denmark

Background: Osteoarthritis $(O A)$ is a heterogeneous disease described by a combination of joint pain, physical disability and radiographic alterations leading to joint failure and total joint replacement (TJR). Commonly used endpoints in OA trials are worsening of pain and joint space narrowing. TJR is normally not considered an endpoint. Age and female gender are considered as major risk factors for developing $O A$

Objectives: We hypothesise that TJR can be used as an endpoint in OA outcome studies within reasonable time frame. To investigate the basis for this hypothesis, we explored the prevalence and incidence of TJR as a reflection of joint failure in the Prospective Epidemiologic Risk Factor (PERF I) study.

Methods: A total of 5,855 Danish postmenopausal women aged 49-88 enrolled in the Prospective Epidemiologic Risk Factor (PERF I) study during 1999-2001 (baseline). Three, six and twelve year follow-up data from the Danish National registry was collected in end of 2014, including occurrence of TJR, OA and other relevant diagnosis. Also, women where at baseline and in 2014 asked whether they had a TJR or OA. The biomarker C1M was measured in baseline serum samples. The PERF I study was carried out in accordance with ICH-GCP and the study protocol was approved by the local ethics committees.

Results: There were 798 women that had their first TJR between baseline and 12 year follow-up; giving an incidence proportion of $13.6 \%$. The TJR women were on average 1 year older $(p=0.010)$ and heavier $(1.7 \mathrm{~kg} / \mathrm{cm} 2, p<0.0001)$, compared to women with no TJR in the follow-up period. The incidence after three and six years, of first ever TJRs, were 171 and 362 corresponding to an incidence proportions of $2.9 \%$ and $6.2 \%$. Next we investigated the TJR incidence rates at 3,6 and 12 years in different subgroup of women: 1) All, PERF I women that experience their first ever TJR (5855); 2) Prior TJR, had a TJR before baseline (266); 3) OA diagnose at baseline but no prior ; TJR (1757) and 4) OA diagnose at baseline and high $\mathrm{C} 1 \mathrm{M}$ ( $>40 \mathrm{ng} / \mathrm{mL}$, median) that after baseline underwent there first ever TJR (841). Group 1 is as described above. The 3, 6 and 12 incidence rates were $9.0,17.3 \%$ and $29.7 \%$ for the prior TJR group, $6.2,12.1 \%$ and $23.3 \%$ for the OA group, and $7.6,13.6 \%$ and $25.1 \%$ for the $\mathrm{OA}+\mathrm{C} 1 \mathrm{M}$ group. The age-dependent prevalence and incidence for the first TJR. The prevalence was insignificant for the age group younger than 60 years old $(<0.1 \%)$. The prevalence increased steadily from the age group 60 to age group 85 ; from $0.1 \%$ to $13.2 \%$.

Conclusions: Within a timeframe of 3,6 or 12 years TJR incidence for women with an OA diagnosis reached $6,12 \%$ and $23 \%$, which was a doubling compared to the Al/population. The incidence increased by adding a single diagnostic measure. This reflects that TJRs are frequent amongst elderly women and that if designed minutiously, such as including specific diagnostic criteria (e.g. biomarker, OA diagnose), it may be feasible to conduct clinical studies with TJR as an endpoint. However, special attention much be directed to the objectiveness of the criteria for TJR. This may build a case for design of outcome studies (joint failure) for developing drugs in OA.

Disclosure of Interest: A. Bay-Jensen Shareholder of: Nordic Bioscience, Grant/research support from: ApproacH (IMI support), Employee of: Nordic Bioscience, C. Bager Employee of: Proscion, A. Bihlet Shareholder of: Nordic Bioscience, Employee of: Nordic Bioscience, C. Thudium Employee of: Nordic Bioscience, I. Byrjalsen Employee of: Nordic Bioscience, H. Nielsen Employee of: Nordic Bioscience, J. Andersen Shareholder of: Nordic Bioscience, Employee of: Nordic Bioscience, B. Riis Shareholder of: Nordic Bioscience, C. Christiansen Shareholder of: Nordic Bioscience, M. Karsdal Shareholder of: Nordic Bioscience, Grant/research support from: ApproacH (IMI support), Employee of: Nordic Bioscience DOI: 10.1136/annrheumdis-2018-eular.7003 